\title{
ESTIMATION OF SERUM VITAMIN D LEVELS AT TERM PREGNANCY IN A TERTIARY CARE CENTRE IN EASTERN INDIA- A CROSS-SECTIONAL STUDY
}

\author{
Monalisha Rajguru1, Irene Ray², Parnamita Bhattacharya3, Somajita Chakraborty ${ }^{4}$ \\ 1 Postgraduate Trainee, Department of Obstetrics and Gynaecology, Medical College, Kolkata. \\ ${ }^{2}$ Senior Resident, Department of Obstetrics and Gynaecology, Medical College, Kolkata. \\ ${ }^{3}$ Assistant Professor, Department of Obstetrics and Gynaecology, Medical College, Kolkata. \\ ${ }_{4}^{4}$ Associate Professor, Department of Obstetrics and Gynaecology, Medical College, Kolkata.
}

\section{BACKGROUND}

ABSTRACT

Vitamin $\mathrm{D}_{3}$ deficiency is very common in pregnant women in India, despite India being a tropical country. Keeping in mind the large percentage of mothers affected by vitamin $\mathrm{D}_{3}$ deficiency, routine antenatal vitamin $\mathrm{D}_{3}$ estimation and supplementation (400IU) may be considered.

This study aims to document incidence of Vitamin $\mathrm{D}_{3}$ deficiency in a cross-section of antenatal mothers and its effects on pregnancy if any.

\section{MATERIALS AND METHODS}

This is a cross-sectional clinical study including a total of 80 mothers with term pregnancy admitted in the antenatal ward of a government hospital in eastern India over a period of 18 months from January 2014 to June 2015. The demographic factors, food habits, Vitamin $\mathrm{D}_{3}$ levels and pregnancy outcomes are documented.

\section{RESULTS}

Vitamin $\mathrm{D}_{3}$ deficiency $(<20 \mathrm{ng} / \mathrm{mL}$ ) is seen in $36.25 \%$ of the population and insufficiency $(20-29 \mathrm{ng} / \mathrm{mL})$ is seen in $38.75 \%$. Vitamin $\mathrm{D}_{3}$ levels are found to be significantly low in multigravida patients from low socio-economic status (BPL card holder) $(\mathrm{p}<0.05)$, as also patients with inadequate antenatal calcium and vitamin D supplementation $(\mathrm{p}=0.000)$. There are not any significant complications in antenatal period attributed to low Vitamin $\mathrm{D}_{3}$ levels. However, people undergoing CS are found to be more Vitamin $\mathrm{D}_{3}$ deficient $\left(\mathrm{p}=0.009\right.$ ). Also, 70 out of 80 mothers took calcium and vitamin $\mathrm{D}_{3}$ supplementation, but still in $72 \%$ of them vitamin $\mathrm{D}_{3}$ level was inadequate. Among the mothers who did not take any supplementation, $90 \%$ showed vitamin $\mathrm{D}_{3}$ deficiency/ insufficiency $(\mathrm{p}=0.000)$. Also, mothers who took lesser amount of total calcium and Vitamin $\mathrm{D}_{3}$ were at significant risk of having vitamin $\mathrm{D}_{3}$ deficiency/ insufficiency $(\mathrm{p}=0.000)$.

\section{CONCLUSION}

Vitamin $D_{3}$ deficiency is very common in antenatal mothers in India and so it might be cost effective to introduce routine estimation of Vitamin $\mathrm{D}_{3}$ as a part of antenatal screen and supplementations should be made widely available as a prophylaxis.

\section{KEYWORDS}

Vitamin $\mathrm{D}_{3}$, Calcium, Deficiency, Pregnancy.

HOW TO CITE THIS ARTICLE: Rajguru M, Ray I, Bhattacharya P, et al. Estimation of serum vitamin D levels at term pregnancy in a tertiary care centre in Eastern India- a cross-sectional study. J. Evolution Med. Dent. Sci. 2017;6(93):6722-6725, DOI: $10.14260 /$ jemds/2017/1455

\section{BACKGROUND}

Vitamin D is a group of fat soluble vitamins found in liver and fish oils. It is an essential component of calcium homeostasis and helps in prevention of rickets in children and osteomalacia in adults. The enzyme responsible for Vitamin D activation, $1 \alpha$ hydroxylase and its receptor have been located in the placenta suggesting a far-reaching role of vitamin D than bone metabolism alone.[1]

$1,25(\mathrm{OH})_{2} \mathrm{D}_{3}$ is the active form of Vitamin D responsible for its endocrine function. However, Vitamin D deficiency is more aptly reflected in the levels of $25(\mathrm{OH}) \mathrm{D}_{3}$ rather than 1 , $25(\mathrm{OH})_{2} \mathrm{D}_{3}$ levels which may be in the normal range or even

'Financial or Other Competing Interest': None.

Submission 19-10-2017, Peer Review 21-11-2017,

Acceptance 27-11-2017, Published 11-12-2017.

Corresponding Author:

Dr. Somajita Chakraborty,

\#83/1/1, Ballygunge Place,

Kolkata-700019, West Bengal, India.

E-mail: somajitac@gmail.com

DOI: $10.14260 /$ jemds $/ 2017 / 1455$ vitamin D deficiency. Also, $25(\mathrm{OH}) \mathrm{D}_{3}$ is more stable and so it is most commonly used to measure Vitamin D levels. The blood level of Vitamin D is tightly regulated by calcium, phosphorus and parathyroid hormone levels.[2]

During pregnancy, serum levels of $1,25(\mathrm{OH}) \mathrm{D}_{3}$ increase upto 2-fold starting at $10-12$ weeks of gestation and reaching a maximum in the third trimester.[3] Due to the increased level of the active form of Vitamin D, pregnant women are likely to have a higher cellular exposure to Vitamin D during the second and third trimesters suggesting a role for Vitamin $\mathrm{D}_{3}$ in obstetric wellbeing. Mothers should have adequate sun exposure as well as proper nutrition during pregnancy for maintaining adequate vitamin $\mathrm{D}_{3}$ levels.

Most cases of Vitamin D deficiency are asymptomatic. ACOG Practice Bulletin, 2011: "Vitamin D: Screening and Supplementation" defines Vitamin D deficiency as $25(\mathrm{OH}) \mathrm{D}_{3}$ levels less than $20 \mathrm{ng} / \mathrm{L}(50 \mathrm{nmol} / \mathrm{L})^{[4]}$ and Vitamin $\mathrm{D}_{3}$ insufficiency as a $25(\mathrm{OH}) \mathrm{D}_{3}$ level of $20-29 \mathrm{ng} / \mathrm{mL}(52.5$ $72.5 \mathrm{nmol} / \mathrm{L}$ ).[5] 
A widespread Vitamin D3 deficiency is seen in pregnant women, necessitating a need to define optimum serum levels of Vitamin $\mathrm{D}_{3}$ and the recommended daily allowance of Vitamin $\mathrm{D}_{3}$ to maintain such optimum levels in these populations. However, more randomised controlled trials should be undertaken to evaluate the advantage and costeffectiveness of routine vitamin $\mathrm{D}_{3}$ estimation among pregnant women and measuring kits should be available at a subsidised rate for this cause.

The purpose of our study is to find out the incidence of vitamin $\mathrm{D}_{3}$ deficiency in mothers at term by estimating vitamin $\mathrm{D}_{3}$ levels (25-OH-vitamin $\left.\mathrm{D}_{3}\right)$ in serum, the correlation between vitamin $\mathrm{D}_{3}$ intake among antenatal mothers and serum Vitamin $\mathrm{D}_{3}$ levels at term and the clinical effects of low Vitamin $\mathrm{D}_{3}$ levels on the course of present pregnancy.

\section{MATERIALS AND METHODS}

A cross-sectional institution-based clinical study was conducted after Institutional Ethics Committee approval, from January 2014 to June 2015 with 80 clinically stable singleton antenatal mothers (aged 18 - 33 yrs.) at term (37+0 $41^{+6}$ weeks) admitted in the antenatal ward of Eden Hospital, Dept. of Obstetrics and Gynaecology. Pregnancy complications like multiple pregnancy, any pre-gestational medical disorder like chronic hypertension, renal insufficiency, bone disease (viz. osteogenesis imperfecta, osteomalacia), malabsorption syndromes, women with skin cancers or skin-related conditions where sun exposure is avoided and mothers of children with rickets were excluded from the study.

Based on systematic random sampling method, a sample size of 76 was calculated. For ease of calculation target sample size was set at 80 subjects. Figure 1 shows summary of study design.

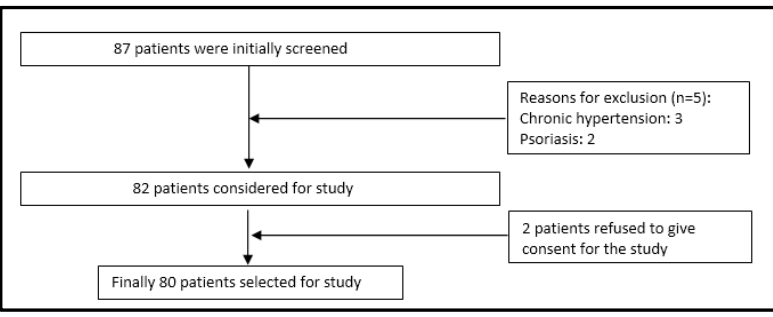

Figure 1. Summary of Study Design

The first subject of study was selected randomly and the other subjects were selected as per systematic random records. Blood samples for Vitamin $\mathrm{D}_{3}$ estimation were sampling method. Detailed case history was taken and clinical examination was done along with checking of antenatal collected from all the patients included in the study. Approximately, $2 \mathrm{~mL}$ of fasting whole blood sample was collected by venepuncture in a non-heparinised tube. The aliquots were then stored in a freezer at $-20^{\circ} \mathrm{C}$ before analysis. Serum 25-hydroxyvitamin $\mathrm{D}_{3}$ concentration was measured by ELISA immunoassay method using an ELISA Reader with Washer. Based on 25-hydroxyvitamin $\mathrm{D}_{3}$ levels, the correlation between Vitamin $\mathrm{D}_{3}$ levels and demographic characteristics and pregnancy outcomes was sought.

\section{RESULTS}

For ease of data representation, all $25(\mathrm{OH}) \mathrm{D}_{3}$ levels have been expressed in a single concentration unit- nanogram per millilitre $(\mathrm{ng} / \mathrm{mL})$ and all Vitamin $\mathrm{D}_{3}$ doses have been expressed in International Unit (IU).

The data was expressed as mean \pm standard deviation, frequency and percentage as applicable. Association between various risk factors and vitamin $\mathrm{D}_{3}$ has been expressed with Odds Ratio. Odds ratio of $<0,0$ and $\geq 1$ indicate - no risk (protective), no association and significant association respectively.

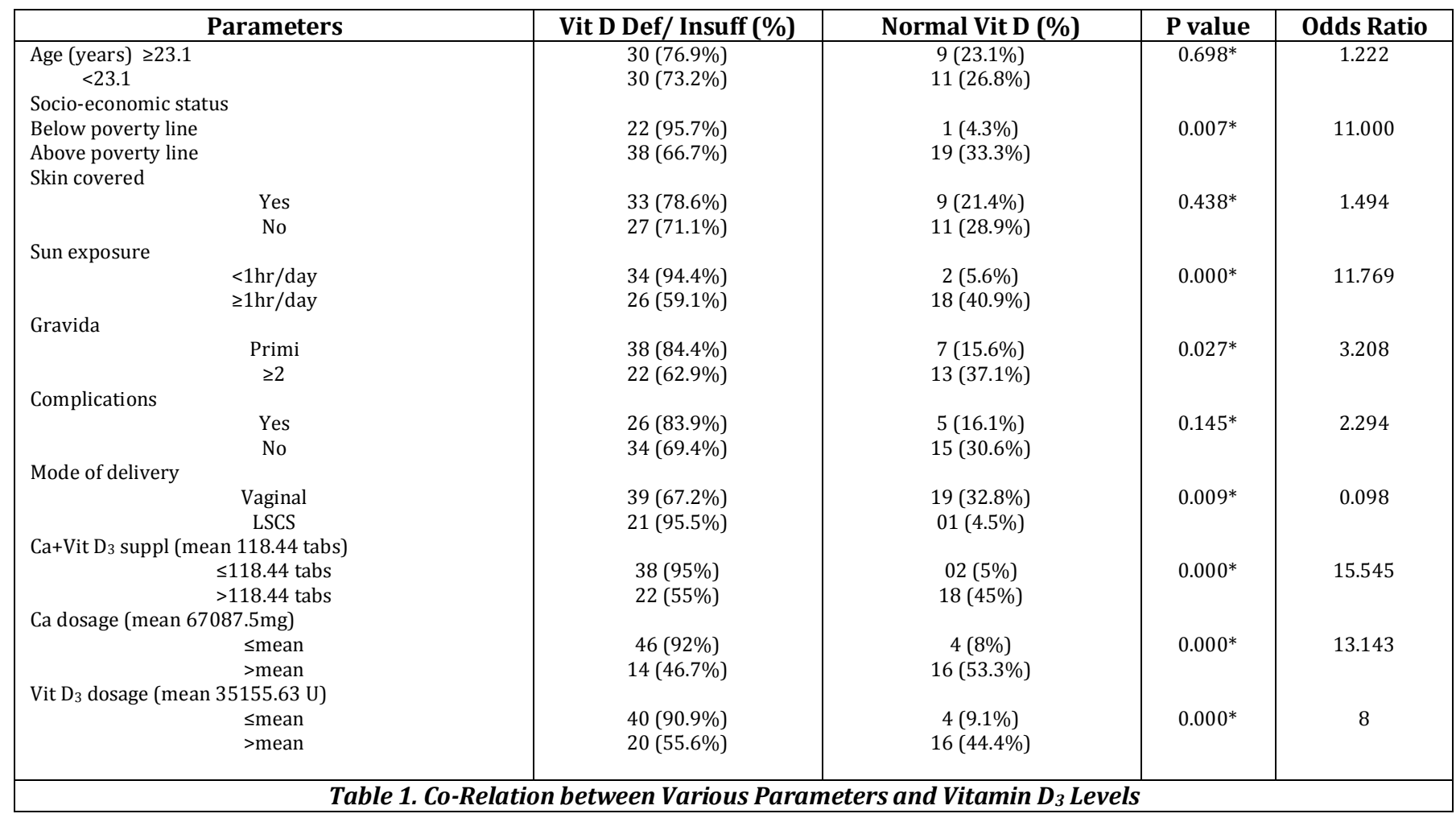


Statistical analysis was carried out using the chi-square test, where two-tailed $\mathrm{p}$ value $<0.05$ was considered as significant. For the data analysis, standard statistical software like Microsoft Excel 2010, SPSS version 20 were utilised.

*Significance calculated using chi-square test.

Table 1 shows that age and clothing habit did not have any significant impact on the vitamin D levels, whereas socioeconomic status, exposure to sun and increasing gravida does seem to significantly influence vitamin D levels. Although, the deficient mothers suffered from various complications like pregnancy induced hypertension, gestational diabetes mellitus, bacterial vaginosis, bone and muscular disorder and caesarean section, none of these associations were statistically significant.

70 out of 80 mothers took calcium and vitamin $\mathrm{D}_{3}$ supplementation $\left(500 \mathrm{mg}\right.$ of calcium and $250 \mathrm{IU}$ of vitamin $\mathrm{D}_{3}$ per tablet), but still in $72 \%$ of them vitamin $\mathrm{D}_{3}$ level was inadequate. Among the 10 mothers who did not take any supplementation, $\quad 90 \%$ showed vitamin $\quad \mathrm{D}_{3}$ deficiency/insufficiency. Thus, mothers not taking calcium and Vitamin $\mathrm{D}_{3}$ tablets were associated with significantly $(\mathrm{p}=0.000)$ reduced serum vitamin $\mathrm{D}_{3}$ level. Also, mothers who took lesser amount of total calcium and Vitamin $\mathrm{D}_{3}$ were at significant risk of having vitamin $\mathrm{D}_{3}$ deficiency/ insufficiency $(p=0.000)$.

\section{DISCUSSION}

Vitamin $\mathrm{D}_{3}$ deficiency is quite rampant these days. However, presently there is insufficient evidence to recommend screening for all pregnant women for vitamin $\mathrm{D}_{3}$ deficiency. During pregnancy, severe maternal vitamin $\mathrm{D}_{3}$ deficiency has been shown to be associated with complications like pregnancy induced hypertension/ pre-eclampsia/ gestational diabetes mellitus/ bacterial vaginosis/ musculoskeletal symptoms. There is a need to raise the desired Vitamin $\mathrm{D}_{3}$ level in pregnancy to $>30 \mathrm{ng} / \mathrm{mL}$ from the conventional $>20$ $\mathrm{ng} / \mathrm{mL}$ as suggested by the Institute of Medicine (IOM), US.

When vitamin $D_{3}$ deficiency is identified, most experts agree that 1000 - 2000 IU per day of vitamin $D_{3}$ is perhaps safe. Recommendations concerning routine vitamin $D_{3}$ supplementation during pregnancy beyond what is contained in prenatal vitamin are controversial.

The present study illustrates the vitamin $\mathrm{D}_{3}$ deficiency statistics among pregnant women, firmly establishing the grave need to tackle this issue urgently.

In our study, the age of the population range between 18 and 33 years. Although, reduced vitamin $\mathrm{D}_{3}$ levels were more in number among the older mothers, no significant relationship has been found between age and vitamin $D_{3}$ levels $(p=0.698)$. Socio-economic status however has been found to have a more significant impact on the vitamin $\mathrm{D}_{3}$ levels. Inadequate vitamin $\mathrm{D}_{3}$ level is more prevalent $(95.7 \%)$ among mothers with lower socio-economic status (below poverty line) compared to higher socio-economic status $(66.7 \%)$ and the difference is statistically significant $(\mathrm{p}=0.007)$.

Pigmentation is cited as an important factor in modulating the levels of $25(\mathrm{OH})_{2} \mathrm{D}_{3}$. Clothing habit of mothers has not been found to be significantly related to vitamin $\mathrm{D}_{3}$ levels. Although, our study shows that covered clothing is 1.4 times associated with reduced serum vitamin $\mathrm{D}_{3}$ levels, but this has no statistical significance $(\mathrm{p}=0.438) . \mathrm{On}$ the other hand, hours of exposure to sun seems to have significant impact on the Vitamin $\mathrm{D}_{3}$ levels. Mothers who had poor sun exposure $(<1 \mathrm{hr} /$ day $)$ are found to have 11 times higher risk of having reduced serum vitamin $\mathrm{D}_{3}$ level, which is statistically significant $(\mathrm{p}=0.000)$.

In our study, 35 out of 80 mothers are primigravida. Among the 45 multigravida ( $G \geq 2$ ) mothers, vitamin $D_{3}$ inadequacy was found in $84.4 \%$ mothers and they are 3 times more prone to develop significantly reduced serum Vitamin $\mathrm{D}_{3}$ level than primigravida $(62.9 \%)(\mathrm{p}=.027)$.

Vitamin $\mathrm{D}_{3}$ deficiency has been shown in various studies to be associated with some complications viz. pregnancyinduced hypertension/ pre-eclampsia/ gestational diabetes mellitus/ bacterial vaginosis/ musculoskeletal symptoms. The distribution of complications in this study are shown in Table 2.

\begin{tabular}{|c|c|c|}
\hline Complications & Number & Percentage \\
\hline PIH/ PET & 15 & 18.75 \\
\hline GDM & 4 & 5 \\
\hline Bacterial vaginosis & 6 & 7.5 \\
\hline Musculoskeletal symptoms & 7 & 8.75 \\
\hline Table 2. Complications Found among the Study Population \\
and their Relative Incidence \\
\hline
\end{tabular}

In this study, $40 \%$ of the mothers are found to have one or more of above complications. Of them, $83.9 \%$ are found to have sub-optimal Vit-D levels, whereas only $69.4 \%$ of the mothers without any of the above complications have low Vitamin $\mathrm{D}_{3}$ levels. Though the complication rate increases with lesser serum vitamin $\mathrm{D}_{3}$ levels, its association is not found to have statistical significance $(\mathrm{p}=0.145)$. This finding is similar to the results of the following studies.

Marya et al suggested that Vitamin D (1200 IU/day) during 32 and 36 weeks of pregnancy was associated with significant reduction of systolic blood pressure (SBP) and diastolic blood pressure (DBP) than non-supplemented group (RR 0.67; 95\% CI 0.33 to 1.35).[6] Parul Singla et al studied 100 pregnant women who received 60,000 IU every fortnight from 28 - 36 weeks of gestation. Vitamin D supplementation during the third trimester of pregnancy was found to be efficacious in reducing the risk of preeclampsia by increasing therapeutic effectiveness of calcium. ${ }^{[7]}$

Three recent case control studies from India, the UK and North Carolina aimed to determine whether early pregnancy $25(\mathrm{OH}) \mathrm{D}$ levels were associated with a later diagnosis of GDM. ${ }^{[8,9,10]}$ Mixed results were obtained. In one study, women with GDM had 2.7 times the odds ratio (CI 1.0 - 7.0) of Vitamin D deficiency in the first trimester as those without. The second showed no association between low Vitamin D levels and a diagnosis of GDM, but did find increased fasting glucose and haemoglobin A1c levels at 28 weeks. The third study found no association between diagnosis of GDM and first trimester Vitamin D deficiency, but had very wide confidence intervals. None of the studies were adjusted for dietary factors or sun exposure. Lau et al showed that $41 \%$ of pregnant women with GDM who lived in Sydney were vitamin D deficient (Lau et al, 2011).

Mothers with vitamin $\mathrm{D}_{3}$ deficiency/ insufficiency are at an increased risk of undergoing caesarean section, because of poor bone quality. In our study, this association was not found to have much significance. $65 \%$ of deficient mothers had vaginal birth and $35 \%$ had caesarean section. Looking at 
the data from another angle, among mothers having vaginal birth $67.2 \%$ were found to have Vitamin $\mathrm{D}_{3}$ deficiency, whereas $95.5 \%$ of the mothers having caesarean section were deficient in Vitamin $D_{3}$. Caesarean section is significantly more common in mothers with deficient or insufficient Vitamin $\mathrm{D}_{3}$ levels $(\mathrm{p}=0.009)$. Odds ratio indicates that vaginal delivery actually protects reduced serum Vitamin $\mathrm{D}_{3}$ level.

From our study, it can be seen that most of the mothers (87.5\%) had taken calcium and vitamin $\mathrm{D}_{3}$ supplementation during pregnancy. Among the mothers who had taken the supplementation $72 \%$ were found to have low vitamin $D_{3}$ levels, whereas $90 \%$ of the mothers who did not take any supplementation had low Vitamin $\mathrm{D}_{3}$ levels (statistically significant, $p=0.000$ ). However, since $72 \%$ of the mothers had low vitamin levels despite calcium and vitamin supplementation, it goes to show that adequate dosage and duration of supplementation is necessary to optimise vitamin $\mathrm{D}_{3}$ levels in our bodies. Indeed, amount of calcium supplementation was found to be directly related to the level of Vitamin $D_{3}$. The mean amount of calcium intake among the study population was found to be $68087.5 \mathrm{mg}$ (each calcium tablet containing $500 \mathrm{mg}$ ). $92 \%$ of the mothers taking less than the mean amount of calcium had vitamin $\mathrm{D}_{3}$ deficiency, whereas only $46.7 \%$ of the mothers taking more than the mean amount of calcium supplementation had low vitamin $\mathrm{D}_{3}$ levels. So lesser amount of total calcium intake is found to be a risk factor for vitamin $\mathrm{D}_{3}$ deficiency/ insufficiency and this correlation is significant $(\mathrm{p}=0.000)$.

In our study, the mean vitamin $\mathrm{D}_{3}$ intake throughout pregnancy in those mothers who took combined calcium and vitamin $\mathrm{D}_{3}$ supplementation was 35155.63 IU (each tablet containing $250 \mathrm{IU}$ ). It was seen that lesser the amount of total Vitamin $\mathrm{D}_{3}$ intake, the more significant is the risk of having vitamin $\mathrm{D}_{3}$ deficiency/ insufficiency. Mothers who took less than the mean amount of vitamin $\mathrm{D}_{3}$ intake had inadequate vitamin $\mathrm{D}_{3}$ levels in $90.9 \%$ cases and those who took more than the mean amount had only a $55.6 \%$ inadequacy $(p=0.000)$. Similar results were reported by Sahu et al[11] and Brooke et al.

However, like most studies, this study also has some limitations. Since our study has included only routine blood tests and blood tests specific for vitamin $\mathrm{D}_{3}$ deficiency, these patients may have some other underlying chronic undiagnosed condition that influences bone metabolism. Serum calcium, serum parathyroid and hepatic hormones could potentially influence calcium and vitamin $\mathrm{D}_{3}$ metabolism in our bodies. Whereas, our study only measured $25(\mathrm{OH}) \mathrm{D}_{3}$ levels which only reflects a low Vitamin $\mathrm{D}_{3}$ level, but may not reflect the exact underlying pathophysiology. Hence, in future, more extensive and in-depth studies (that include measurement of all parameters influencing vitamin $\mathrm{D}_{3}$ levels in our bodies) should be undertaken.

\section{CONCLUSION}

Our study showed inadequate vitamin $\mathrm{D}_{3}$ levels in both symptomatic and asymptomatic mother who got admitted in the duration of the study. So, routine vitamin $\mathrm{D}_{3}$ estimation among pregnant women may be introduced as a means of safe-guarding this at-risk population and measuring kits should be made available at government sectors at a subsidised rate for this cause. Also like iron supplementation in developing countries, vitamin $\mathrm{D}_{3}$ (400 IU) could also be routinely prescribed for the better health of mother and baby.

\section{REFERENCES}

[1] Mulligan ML, Felton SK, Riek AE, et al. Implications of vitamin D deficiency in pregnancy and lactation. Am J Obstet Gynecol 2010;202(5):429. e1-e9.

[2] Christakos S, Ajibade DV, Dhawan P, et al. Vitamin D: metabolism. Endocrinol Metab Clin North Am 2010;39(2):243-53.

[3] Brannon PM, Picciano MF. Vitamin D in pregnancy and lactation in humans. Annu Rev Nutr 2011;31:89-115.

[4] ACOG Committee Opinion No. 495: Vitamin D: screening and supplementation during pregnancy. Obstet Gynecol 2011;118(1):197-8.

[5] Chung M, Balk EM, Brendel M, et al. Vitamin D and calcium: a systematic review of health outcomes. Evidence Report/Technology Assessment (Full Rep) 2009;183:1-420.

[6] Marya RK, Rathee S, Manrow M. Effect of Calcium and Vitamin D supplementation on toxaemia of pregnancy. Gynecologic and Obstetric Investigation 1987;24(1):38-42.

[7] Singla P, Parkash AA, Lal H. et al. Benefits of Vitamin D supplementation in pregnancy for prevention of preeclampsia. International Journal of Pharmacy \& Biological Sciences 2012;2(3):144-50.

[8] Zhang C, Qiu C, Hu FB, et al. Maternal plasma 25Hydroxyvitamin D concentrations and the risk for gestational diabetes mellitus. PLoS One 2008;3(11):e3753.

[9] Baker AM, Haeri S, Camargo CA, et al. First-trimester maternal vitamin D status and risk for gestational diabetes mellitus (GDM): a nested case-control study. Diabetes Metab Res Rev 2012;28(2):164-8.

[10] Makgoba M, Nelson SM, Savvidou M, et al. Firsttrimester circulating 25-Hydroxyvitamin D levels and development of gestational diabetes mellitus. Diabetes Care 2011;34(5):1091-3.

[11] Sahu M, Das V, Aggarwal A, et al. Vitamin D replacement in pregnant women in rural north India: a pilot study. European Journal of Clinical Nutrition 2009;63(9):1157-9. 